The risk of stillbirth increased monotonically with increasing FPG, suggesting no biological justification for one GDM diagnostic threshold over another.

Conclusion Women with raised FPG experience higher risk of late stillbirth. If diagnosed with GDM and managed accordingly, this appears to be largely mitigated. Inconsistent diagnostic practices however leave many women with borderline hyperglycaemia exposed to higher risks of stillbirth.

\section{OP81 A MULTI-OMICS APPROACH TO INVESTIGATE THE INFLAMMATORY RESPONSE OF LIFE COURSE SOCIOECONOMIC POSITION: FINDINGS FROM EPIC-ITALY}

${ }^{1} \mathrm{R}$ Castagné*, ${ }^{1} \mathrm{M}$ Kelly-Irving, ${ }^{2} \mathrm{SA}$ Kyrtopoulos, ${ }^{3,4} \mathrm{P}$ Vineis, ${ }^{3} \mathrm{M}$ Chadeau-Hyam, ${ }^{1}$ C Delpierre. 'LEASP, UMR 1027, Inserm-Université Toulouse III Paul Sabatier, Toulouse, France; ${ }^{2}$ National Hellenic Research Foundation, Institute of Biology, Pharmaceutical Chemistry and Biotechnology, Athens, Greece; ${ }^{3}$ Department of Epidemiology and Biostatistics, Imperial College, MRC-PHE Centre for Environment and Health, School of Public Health, London, United-Kingdom; ${ }^{4}$ Molecular and Genetic Epidemiology Unit, Italian Institute for Genomic Medicine (IIGM), Torino, Italy

\subsection{6/jech-2019-SSMabstracts.84}

Background Lower socioeconomic position (SEP) has consistently been associated with poorer health. Chronic inflammation has been proposed as having a prominent role in the construction of social inequalities in health. Disentangling the effects of social disadvantage along the life course on inflammation is key in elucidating biological mechanisms underlying socioeconomic disparities. In this study we investigate how life course socioeconomic conditions influence omics measures of inflammation at different molecular level traits from a subset of 173 Italian participants of the European Prospective Investigation into Cancer and Nutrition (EPIC) cohort.

Methods We used genome-wide methylation and transcriptional profiles obtained from blood samples from 178 Italian participants of the EPIC cohort. Starting from 824 genes involved in human inflammatory responses and corresponding to $11502 \mathrm{CpG}$ sites, we first identified 61 potential cis acting $\mathrm{CpG}$ loci whose degree of methylation was associated with gene expression (eMS) at a Bonferonni correction, out of which $78.7 \%$ were inversely associated with gene expression in cis. We further investigate the relationships between indicators of SEP at 3 life stages through father's occupation, education and highest household occupation and the 61 cis eMS, involved in inflammation and functionnally relevant, separately and combined through an inflammatory methylome score. We finally investigated life course effects of early-life SEP experiences by sequentially controlling for time-ordered SEP.

Results Our results consistently show that participants with a less advantaged SEP in young adulthood or in adulthood exhibit, later in life, a lower inflammatory methylome score ( $\beta=-0.0075$, P-value $=0.0067, \beta=-0.0076, P$-value $=0.0073$ for educational level and highest household occupational position respectively), hence suggesting an overall increased level of expression for the corresponding inflammatory-related genes. Adjusting for either behavioural factors (smoking status, alcohol consumption and physical activity) and bmi, or all of them together only marginally affected our results: effect size estimates showed consistent signs, and associations reach statistical significance $(\mathrm{P}<0.05)$ for both participant's education and highest household occupational position. Adopting a life course approach weakened these associations suggesting a common pathways between young and later in life SEP. Sensitivity analyses indicated that our findings were not affected by the way the inflammatory methylome score was calculated.

Conclusion Our results support the hypothesis that social inequalities impacts, independently from behavioural factors, adult physiology through inflammation and can be observed at the DNA methylation level. Understanding biological mechanisms by which social environment influences the inflammatory system has important implications in treatment and especially in prevention, by potentially identifying modifiable factors in the environment that affect physiological health.

\section{OP82 DOES A RAPID REVIEW VERSION OF A LARGE EPIDEMIOLOGICAL SYSTEMATIC REVIEW FAIL TO IDENTIFY MANY ELIGIBLE STUDIES, AND WHAT IMPLICATIONS DOES THIS HAVE FOR THE RESULTS OF THE REVIEW?}

M Barnish*. Evidence Synthesis and Modelling for health Improvement (ESMI), University of Exeter, Exeter, UK

\subsection{6/jech-2019-SSMabstracts.85}

Background Systematic reviews (SR) are the gold standard evidence synthesis method. Rapid reviews (RR) have been proposed as an alternative method that may provide evidence in a more timely fashion to inform clinical decision making and policy making. However, RR may fail to identify all relevant evidence, which may bias the review conclusions. An analysis was conducted to compare SR and RR versions of a large epidemiological review in terms of completeness and efficiency of evidence retrieval and any differences in overall review findings.

Methods A SR on the political determinants of health was conducted with searches in November 2017 on 10 scholarly bibliographic databases using a combination of $\mathrm{MeSH}$ terms and key words, accompanied by a search on Google Scholar (GS) and backward citation chasing. Internationally comparative studies assessing the relationship between any of four political themes (democracy, globalisation, political tradition, and welfare state) and any population health outcome, excluding healthcare expenditure, were eligible for inclusion. A RR version of this review was conducted with the same search dates. The RR comprised a GS search for health plus each of 'politics', 'political' and the four political themes plus backward and forward citation chasing. The SR and RR were compared on completeness ( $\%$ of total included studies identified), efficiency ( $\%$ of reviewed records that were included) and results profile (\% of included studies with positive vs non-positive results). Analysis was descriptive in terms of $\mathrm{n}(\%)$ and used chi-square and McNemar test as appropriate in SPSS v.25.

Results 114 studies were eligible for inclusion, of which SR identified 101 (89\%, due to absence of forward citation chasing) and RR 64 (56\%, McNemar test $\mathrm{p}<0.001)$. SR reviewed 35,262 records $(0.3 \%$ were included $)$ and $\mathrm{RR}$ reviewed 92 records $(70 \%$ were included). For the welfare state theme, 54 (77\%) studies had positive results in SR vs $31(78 \%)$ in RR (chi-square $=0.002, \mathrm{p}=0.966$ ), for political tradition theme 3 $(60 \%)$ vs $2(50 \%$, chi-square $=0.090, p=0.764)$, for democracy theme $14(78 \%)$ vs $14(82 \%$, chi-square $=0.114, p=0.735)$, and for globalisation theme $3(17 \%)$ vs $5(38 \%$, chi-square $=1.873$, $\mathrm{p}=0.171)$. 
Conclusion RR identified significantly fewer included studies than SR, but there were no significant differences in the review results. RR offered greater efficiency with far greater $\%$ of reviewed records being included. This analysis benefited from using data from a large scale epidemiological review. However, it only assessed one broad topic area. Further research and evidence synthesis is needed to assess the value of RR in an epidemiological setting.

\section{Mental Health 2}

\section{OP83 HOW MUCH OF THE EFFECT OF DISABILITY ACQUISITION ON MENTAL HEALTH IS MEDIATED THROUGH EMPLOYMENT AND INCOME? A CAUSAL MEDIATION ANALYSIS QUANTIFYING INTERVENTIONAL INDIRECT EFFECTS USING LONGITUDINAL DATA FROM WORKING AGE AUSTRALIANS}

${ }^{1} \mathrm{Z}$ Aitken*, ${ }^{1} \mathrm{JA}$ Simpson, ${ }^{1} \mathrm{R}$ Bentley, ${ }^{1,2} \mathrm{~T}$ Blakely, ${ }^{1} \mathrm{AM}$ Kavanagh. ${ }^{1}$ Melbourne School of Population and Global Health, The University of Melbourne, Melbourne, Australia; ${ }^{2}$ Department of Public Health, The University of Otago, Wellington, New Zealand

10.1136/jech-2019-SSMabstracts.86

Background There is evidence that disability acquisition causes a decline in mental health, but few studies have examined the causal mechanisms through which the effect operates. Our previous research examined three broad groups of socioeconomic characteristics and found that material factors, rather than psychosocial or behavioural, predominately mediated the effect. However, there remains a lack of understanding about which specific material factors drive the association, limiting the development of effective interventions to improve the mental health of people with disabilities. This study examined how much changes to employment and income mediate the effect of disability acquisition on mental health.

Methods We used data from the Household, Income and Labour Dynamics in Australia Survey, a longitudinal study of Australian households, which collects information annually on a wide range of demographic, social, economic, and health characteristics. We used four waves of data (2011-2014) to compare self-reported mental health between working aged individuals who acquired a disability $(n=233)$ and those who remained disability-free $(n=5419)$. Mental health was measured using the mental health inventory, a subscale of the SF36 health questionnaire. We conducted a causal mediation analysis to quantify interventional indirect effects of disability acquisition on mental health operating through two distinct mediators: employment status and household income. This novel method enables quantification of path-specific effects operating through multiple mediators. We used multiple imputation with 50 imputed datasets to account for missing data and conducted analyses in Stata/SE 15.

Results Disability acquisition was estimated to cause a fivepoint decline in mental health (estimated mean difference: $-4.8,95 \%$ CI $-7.0,-2.7)$. The interventional indirect effect through employment was estimated to be a 0.5 point difference $(-0.5,95 \%$ CI $-1.0,0.0)$, accounting for $11 \%$ of the total effect, whereas there was no evidence that income explained any of the effect.

Conclusion Using a novel approach to mediation analysis which addresses limitations of traditional mediation methods and can be estimated under weaker assumptions than natural indirect effects, we found that some of the effect of disability acquisition on mental health was explained by changes to employment, but not income. Key limitations included strong assumptions about unmeasured confounding and the small number of people acquiring a disability in our dataset. The findings have important policy implications, highlighting the need to improve employment opportunities and vocational training for people who acquire a disability to improve mental health and reduce current mental health inequalities experienced by people with disabilities.

\section{OP84 COULD INCREASING INCOME IN LONE-PARENT HOUSEHOLDS REDUCE POPULATION PREVALENCE AND INEQUALITIES IN CHILDREN'S MENTAL HEALTH PROBLEMS? A POLICY SIMULATION IN THE UK MILLENNIUM COHORT STUDY}

${ }^{1} S$ Hope ${ }^{*},{ }^{2}$ A Pearce, ${ }^{1} \mathrm{R}$ Viner, ${ }^{3} \mathrm{~S}$ Morris, ${ }^{4} \mathrm{D}$ Taylor-Robinson, ${ }^{1} \mathrm{H}$ Roberts, ${ }^{1} \mathrm{~S}$ Chigogora. ${ }^{1}$ Population, Policy and Practice Programme, University College London, London, UK; ${ }^{2} \mathrm{MRCI}$ CSO Social and Public Health Sciences Unit, University of Glasgow, Glasgow, UK; ${ }^{3}$ Centre of Applied Health Research, University College London, London, UK; ${ }^{4}$ Department of Public Health and Policy, University of Liverpool, Liverpool, UK

\subsection{6/jech-2019-SSMabstracts.87}

Background About half of lone-parent families in the UK live in relative poverty (income below $60 \%$ of the national median) compared to a quarter of two-parent households, and family hardship is associated with poorer child mental health. There is little evidence on whether reducing income differences between lone- and two-parent households could reduce inequality in child mental health problems (CMHP). Using data from the UK Millennium Cohort Study ( 18000 children born 2000-2002), we investigated whether equalising income between lone- and two-parent households could reduce prevalence and inequality in CMHP. We also simulated achievement of a government target to reduce child poverty to less than $10 \%$ of the population (Child Poverty Act 2010), to assess whether tackling low income could reduce inequality in CMHP related to family structure.

Methods Exposure was family structure (lone- or two-parent household) when the child was $9 \mathrm{~m}$; mediator was equivalised household income per week at 3y; outcome was parent-report CMHP at 5y (Strengths and Difficulties Questionnaire; borderline-abnormal, yes/no). We accounted for attrition to MCS at $5 \mathrm{y}$, and baseline and intermediate confounders. Analyses were carried out in an analytic sample of 11,193.

We estimated the controlled direct effect of family structure on CMHP in logistic marginal structural models, weighted for attrition, and adjusted for confounding and mediation by household income. Prevalence was assessed overall and according to family structure; inequalities were computed using risk ratios (RRs) and differences (RDs) [95\% CIs]. We re-estimated the controlled direct effect after increasing income according to two simulations. In the first, we equalised income between lone- and two-parent households. In the second, we modelled achievement of the child poverty target by increasing income for some lone-and two-parent families, reducing the proportion of households living in relative poverty to less than $10 \%$. Results Prevalence of CMHP was 8.5\% [7.6, 9.5], and children from lone-parent households were more likely to exhibit poorer mental health (RR 1.73; RD 5.70). Equalising income between lone- and two-parent households reduced prevalence $(8.2 \%[7.3,9.0])$, and inequality (RR, 1.37; RD, 2.86) in 